\title{
Un barrage contre le Pacifique
}

Polders et développement au Cambodge

Pierre-Yves Le Meur

\section{OpenEdition \\ Journals}

Édition électronique

URL : http://journals.openedition.org/anthropodev/364

DOI : 10.4000/anthropodev.364

ISSN : 2553-1719

Éditeur

APAD - Association pour l'anthropologie du changement social et du développement

Édition imprimée

Date de publication : 1 septembre 2015

Pagination : 27-58

ISBN : 979-10-93476-02-5

ISSN : 2276-2019

\section{Référence électronique}

Pierre-Yves Le Meur, « Un barrage contre le Pacifique », Anthropologie \& développement [En ligne],

42-43 | 2015, mis en ligne le 01 décembre 2016, consulté le 19 avril 2019. URL : http://

journals.openedition.org/anthropodev/364 ; DOI : 10.4000/anthropodev.364

La revue Anthropologie \& développement est mise à disposition selon les termes de la Licence Creative Commons Attribution 4.0 International. 


\title{
Un barrage contre le Pacifique : polders et développement au Cambodge ${ }^{1}$
}

\author{
Pierre-Yves Le Meur
}

Le texte analyse un projet de réhabilitation d'aménagements hydrauliques concernant une zone de 11000 hectares de polders situés au sud du Cambodge. Le projet comprend aussi un important volet de transfert de gestion des polders à une "communauté d'usagers des polders", un travail de régularisation des droits fonciers, ainsi qu'un appui à la production agricole et au crédit rural, dans une logique de développement intégré. Le transfert de gestion et le volet foncier vont donner au projet son identité et son caractère " expérimental " puis "pilote ", alors même qu'ils n'étaient pas clairement affichés au démarrage de l'action. L'analyse montre comment certains mots - communauté, projet pilote, transfert de gestion, participation - et leurs supports - écrits, discours, actions contribuent à forger une mémoire institutionnelle et une gouvernementalité spécifiques via une intervention dont les raisons se construisent dans le cours de l'action et non pas en amont de celle-ci. L'approche combinant sociologie de la traduction et anthropologie du développement permet d'explorer les relations mutuellement constitutives entre intervention de développement et politique publique pour se poser in fine la question du "monde commun " qui est ainsi produit.

The text analyses a hydraulic rehabilitation project covering an area of 11,000 hectares of polders in South Cambodia. Based on a logic of integrated development, the project also included the transfer of management to a 'water user community', the regularization of land rights, agricultural extension and rural credit. The inclusion of management transfer and land titling in the project, despite the fact that these elements were not explicitly stated from the outset,

${ }^{1}$ Je remercie ici chaleureusement Jean-Pierre Jacob et Jean-Pierre Olivier de Sardan pour leurs lectures avisées, tout comme pour leurs taquineries amicales. Les erreurs et limites restent miennes. Quant au titre, il est emprunté au livre de Marguerite Duras dont l'action se joue à l'endroit décrit dans ce texte (Duras, 1950). 
gave it an 'experimental' character and, later also, a 'pilot' status. The analysis shows how certain words - community, pilot project, management transfer, participation - and their inscription in texts, discourses and actions contributed to the crafting of an institutional memory and a specific form of governmentality in the context of an intervention, whose rationale was constructed over the course of the project rather than beforehand. A combination of the sociology of translation and the anthropology of development enables the exploration of the mutually constitutive relations between development intervention and public policy so as, finally, to raise the question regarding the 'common world' produced as a result.

\section{Introduction}

On pourrait, en référence au travail fondateur de Maurice Halbwachs sur la mémoire collective (1950), avancer que le dispositif du développement favorise la construction d'une mémoire faiblement collective ou du moins extrêmement fragmentée du fait de la tension entre une oralité peu partagée et une écriture très formalisée, qui laisse peu de place à la constitution $d^{\prime}$ un espace public ${ }^{2}$. Entre mémoire et oubli émergent des thèmes connexes, qui sont ceux du malentendu, parfois productif ${ }^{3}$, de la mésentente, et donc, en creux, de la construction d'un monde commun qui pourrait constituer l'horizon du développement.

"Par mésentente, on entendra un type déterminé de situation de parole : celle où l'un des interlocuteurs à la fois entend et n'entend pas ce que dit l'autre. " (Rancière, 1995 : 12)

Rancière distingue la mésentente de la méconnaissance, qui « suppose que l'un ou l'autre des interlocuteurs ou les deux [...] ne sachent pas ce qu'il dit ou ce que dit l'autre ", et du malentendu, qui "repose sur l'imprécision des mots " (ibid.: 12). II précise que la méconnaissance peut résulter

\footnotetext{
${ }^{2}$ L'absence d'espaces publics de diffusion de l'information et de négociation (Strukturen von Nichtöffentlichkeit) constitue une caractéristique structurelle de nombreuses situations de projets de développement (Bierschenk, Elwert \& Kohnert, 1993: 32-33).

3 Paul Bohannan parlait en 1958 de " working misunderstanding »; je remercie Jean-Pierre Jacob pour avoir attiré mon attention sur cette référence.
} 
"d'une simple ignorance, d'une dissimulation concertée ou d'une illusion constitutive " (ibid.), ce qui nous rapproche de l'analyse du développement comme "système d'ignorance" (Hobart, 1993). Rancière différencie ensuite la mésentente du " différend " (Lyotard, 1983) :

"La mésentente ne concerne pas la question de l'hétérogénéité des régimes de phrases et de la présence ou de l'absence d'une règle pour juger des genres de discours hétérogènes. Elle concerne moins l'argumentation que l'argumentable, la présence ou l'absence d'un objet commun entre un $X$ et un $Y$. Elle concerne la présentation sensible de ce commun, la qualité même des interlocuteurs à le présenter. » (ibid. : 14)

En ce sens, la mésentente est consubstantielle à la politique : "La politique est d'abord le conflit sur l'existence d'une scène commune, sur l'existence et la qualité de ceux qui y sont présents. " (ibid. : 49)

La politique est aussi, si l'on suit les propositions de Latour, non pas un domaine distinct de la société, mais un mode d'action et de connaissance, un régime d'énonciation particulier qui vise à construire un collectif, un " monde commun » à partir d'éléments disparates (Latour, 2002, 2008).

C'est l'angle d'attaque que j'utiliserai pour comprendre le monde du développement - dans une de ses expressions localisées, un projet au Cambodge-, un monde comme un autre, qui produit ses théories, entre autres en mobilisant des éléments épars empruntés à d'autres champs sociaux et disciplinaires : la recherche, la religion, la politique, la pédagogie, l'entreprise, etc. C'est en ce sens que l'on peut qualifier le développement de constructivisme (le terme de bricolage aurait aussi pu faire l'affaire, en première approximation) : les éléments qui le composent génèrent et véhiculent des concepts, des théories, des représentations, des catégories, qui s'endogénéisent au travers de leurs usages, au-delà de leur origine souvent hybride et généralement oubliée. On retrouve ici, via la question de la construction d'un monde commun qui englobe les dimensions de la mésentente, du malentendu, de la méconnaissance et du différend, l'enjeu de la mémoire institutionnelle, de la manière dont ce monde particulier gère son rapport aux temporalités plurielles qui contribuent à le constituer. 
Dans une première partie je reviendrai rapidement sur les discontinuités et malentendus au fondement de la construction du développement. L'étude de cas d'un projet au Cambodge qui constitue le cœur de ce texte, montrera comment certains mots - communauté, projet pilote, transfert de gestion, participation - et leurs supports - écrits, discours, actions contribuent à forger une mémoire institutionnelle et une gouvernementalité spécifiques via des interventions dont les raisons se construisent dans le cours de l'action et non pas en amont de celle-ci. On se posera en fin de compte la question de savoir s'il s'agit bien de la construction d'un monde commun (mais commun à qui exactement ?) émergeant autour des réinterprétations multiples des enjeux du développement et de malentendus productifs, ou de la reconduction d'une non-rencontre, d'une mésentente toujours répétée, que la profusion de récits concurrents symboliserait ?

\section{La construction du développement : récits, malentendus et discontinuités}

Le développement comme constructivisme - i.e. construction traversée d'idéologie (Le Meur, 2008a) - produit simultanément un monde et un savoir sur ce monde, selon une logique qui ressemble fort à la logique coloniale que Peter Pels (1996) qualifie de "pidginisation "4. Pels montre que l'ethnographie administrative mise en œuvre par les fonctionnaires coloniaux et leurs intermédiaires locaux n'est pas simple dévoilement d'un monde à gérer mais collaboration créative entre administrateurs et leaders locaux lors de leurs interactions, mais dont les effets sur les deux mondes dépassent ces seuls moments (ibid. : 749-753; voir aussi Hannerz, 1987) ${ }^{5}$.

\footnotetext{
${ }^{4}$ Dans une étude des interactions entre les langages du gouvernement indirect et de la politique locale dans les monts Uluguru de l'est du Tanganyika des années 1920 et 1930. La question de "l'ethnographie administrative » que traite Pels dans ce même texte renvoie à un débat sur la connaissance ou l'ignorance des administrateurs quant à leurs circonscriptions et sur les rapports complexes entre savoir ethnographique, représentations et pratique administrative à l'époque de la colonisation (voir Spittler, 1981 ; Pels, 1997 ; Lentz, 2006 : 72). On peut repérer de nombreux échos de ce débat dans l'univers du développement (voir par exemple Hobart, 1993).

${ }^{5}$ On peut voir dans l'extraordinaire succès de la loi de 1901 sur les associations à but non lucratif dans l'Afrique postcoloniale un exemple abouti de pidginisation touchant non seule-
} 
L'idée d'une collaboration créative n'est pas synonyme d'accord et de transparence, et la rencontre entre administrateurs coloniaux et leaders locaux se construit pour une large part autour de malentendus productifs, d'une appropriation sélective de leurs contributions respectives à la situation d'interface qu'ils construisent, et donc aussi d'un voile mis sur d'autres aspects - acteurs et processus - qui vont rester en dehors de la rencontre. L'interface est, ici comme ailleurs, faite de liens et de discontinuités, de logiques à la fois "enchevêtrées " (Olivier de Sardan, 2001) et segmentées ${ }^{6}$. Si l'on poursuit dans la ligne de Jacques Rancière, on se situe quelque part entre le malentendu (la polysémie autorisant le dialogue), la mésentente (l'écoute mutuelle est toute relative) et la méconnaissance, éventuellement entretenue par le dispositif de reporting de l'aide et le jeu $d^{\prime}$ intermédiaires et de courtiers ${ }^{7}$.

Les discontinuités ont une dimension cognitive dont la mise en forme passe par la mobilisation de concepts comme ceux que nous avons brièvement abordés ci-dessus. Pour dire les choses autrement, connaissance et ignorance sont les deux faces d'un même phénomène et la discussion sur la validité des notions employées dans des interventions - lignage, village, consensus, communauté, participation, gouvernance, ONG, société civile... - ne vise pas la question de leur vérité, par exemple sur la base d'une opposition entre concepts scientifiques et notions relevant de la pratique administrative ou développementiste, simplification trop souvent à l'œuvre dans les approches populistes du développement (Hobart, 1993, par exemple). Il s'agit plutôt de décrire comment des mots et des idées aux

ment les organisations paysannes mais aussi une vaste gamme d'associations culturelles et également religieuses.

${ }^{6}$ Comme l'écrit Hannah Arendt, " partout où des hommes se rassemblent, que ce soit dans la sphère privée ou sociale ou politico-publique, un espace se crée qui les rassemble et simultanément les sépare » (1995: 59).

7 On pourrait aussi utiliser la notion de « cadrage » proposée par Michel Callon (1999 : 405412), qui correspond à l'ensemble des éléments humains ou non humains contribuant à délimiter l'espace du jeu et des interactions entre acteurs, notion qu'il lie à celle de " débordement " qui permet de penser les changements dans le jeu des éléments internes nécessaires au cadrage. Je remercie Jean-Pierre Jacob pour avoir attiré mon attention sur ce rapprochement. 
trajectoires complexes en viennent à endosser un rôle actif dans la construction des situations de développement. Ce sont des " non construits " qui constituent en quelque sorte la grammaire (Lemieux, 2009) du constructivisme développementiste ${ }^{8}$. Ces mots et ces idées sont inséparablement opérateurs de visibilisation et d'invisibilisation d'acteurs, de groupements, d'intérêts, de savoirs, de processus (Hobart, 1993 ; Scott, 1998 ; Le Meur, 2007 : 293-294). Considérés dans une durée plus longue, ils participent des formes de l'institutionnalisation analysées par Mary Douglas dans leurs dimensions inséparablement normatives et cognitives : "Institutions remember and forget » (1987:69).

Les études empiriques des situations de développement montrent aussi que, derrière le métarécit du développement invoqué à l'envi par les anthropologues déconstructionnistes ou "néo-dépendantistes" (Bierschenk, 2008), se fabrique une multitude de micro-récits portés par des acteurs sociaux (agrégés avec plus ou moins de force en " groupes stratégiques ") dont les intérêts, les ressources et les représentations sont divers (Sivaramakrishnan, 2000). Elles montrent les interventions de développement comme entrelacs d'histoires partielles, mises en récit conjoncturelles, reprises inédites de mots passés de mode, sélection de relations causales et chaînes de traduction ${ }^{9}$, dispositifs au sens de réseaux hétéro-

${ }^{8}$ Je remercie Jean-Pierre Jacob pour cette remarque.

${ }^{9}$ Le terme de traduction est ici employé dans le sens que lui donne Michel Callon, pour qualifier un processus constitué d'étapes qui peuvent éventuellement se chevaucher : la " problématisation " comme " entre-définition des acteurs " et " définition de points de passages obligés ", les dispositifs d' « intéressement " visant à la construction d'alliances, l'« enrôlement" ou modalité de coordination des rôles, et la "mobilisation " des alliés, posant en particulier la question de la représentativité des " porte-parole » (Callon, 1986 : 180 et sq. ; Callon et al., $2001: 73$ et sq.). La notion de traduction ainsi définie s'applique à des séries événementielles diverses : innovation technologique (Latour, 1992), travail scientifique (Latour, 1987), mise en œuvre de politique publique ou de projet de développement (Callon et al., 2001; Mosse, 2005 ; Le Meur, 2006, 2011). Elle s'inscrit dans le cadre de la théorie de l'acteur réseau qui s'appuie sur trois principes (Callon et al.. : 175-177) : (i) l'agnosticisme de l'observateur qui ne privilégie aucun point de vue et par conséquent embrasse dans une même perspective des processus qui seront à un moment donné conçus comme des "succès » ou des " échecs »; (ii) un " principe de symétrie généralisée » quant au rôle et au poids des acteurs humains et non-humains (englobés dans la notion d'actant empruntée à la sémiologie de Greimas) ; (iii) un principe de « libre-association » qui exige que 
gènes d'acteurs, instruments et idées, pratiques discursives légitimantes: on se situe là plus près du développement en train de se faire, via ses multiples avatars et localisations et ses jeux complexes d'acteurs et d'institutions.

Différentes strates conceptuelles tendent ainsi à s'accumuler selon un processus de sédimentation qui transforme la nature des notions qui soustendent les interventions et facilite l'oubli de leur ancienneté, tout en laissant à l'observateur attentif la possibilité de repérer leurs origines. L'image de la sédimentation est toutefois insuffisante, car ces strates peuvent aussi jouer simultanément, chaque intervention tendant à activer une forme ou une autre de catégorisation et de découpage de la réalité, provoquant en retour des mouvements locaux de réinterprétation et réaménagement de ces conceptualisations des entités sociales ${ }^{10}$.

\section{Polders, projet et politique}

L'exemple qui suit nous permet d'explorer plus avant le caractère mutuellement constitutif de la relation entre intervention et politique que David Mosse analyse dans un ouvrage consacré à un projet de développement spécifique et dont les catégories de projet-pilote, de transfert de gestion et de participation constituent des opérateurs clefs. Mosse articule son point de vue sur cinq propositions qui relèvent d'une approche en termes d'acteur-réseau et partent de l'idée que les politiques remplissent une fonction de légitimation plutôt que d'orientation de l'action :

"(1) Policy primarily functions to mobilise and maintain political support, that is to legitimise rather than to orientate practice.

"l'observateur abandonne toute distinction a priori entre faits de Nature et faits de Société » (ibid. : 176).

${ }^{10}$ Voir Sivaramakrishnan $(2000: 448)$ : « Governmental procedures that nominate one form of community as relevant to the government's vision of development promptly move villagers and political representatives to reveal the existence of numerous other forms of community. The denial of other forms of community, implicit in the imposition of one form, threatens certain interests and identities. These are then asserted in the ensuing contest to give shape to public order institutions $"$. 
(2) Development interventions are not driven by policy but by the exigencies of organisations and the need to maintain relationships.

(3) Development projects work to maintain themselves as coherent policy ideas (as systems of representations) as well as operational systems. (4) Projects do not fail; they are failed by wider networks of support and validation. (5) 'Success' and 'failure' are policy-oriented judgements that obscure project effects. ॥ (Mosse, 2005 : 14-20)

La gestion de la tension entre le fonctionnement du projet comme isolat (relatif) technique, économique et institutionnel et la nécessité de l'insérer dans un réseau d'alliances plus large à des fins de légitimation et donc de pérennisation se situe au cœur de l'analyse.

\section{Contexte}

Le Cambodge, ancien protectorat français puis pays allié parfois malcommode des Occidentaux après son indépendance en 1953, a subi à partir de 1975 la dévastation du régime génocidaire khmer rouge (Chandler, 1991 ; Kiernan, 2002 ; Hinton, 2005) avant d'être libéré par l'armée vietnamienne fin 1978. Après dix années de reconstruction dans un contexte de fort isolement international et de poursuite, appuyée par la Chine et les USA, de la guérilla khmer rouge (Frings, 1997; Gottesman, 2002 ; Slocomb, 2003), le retrait de l'armée vietnamienne et les accords de Paris de 1993 ouvrent la porte au dispositif de l'aide internationale. On note en particulier l'intervention très controversée de l'UNTAC (Chong, 2002) et la floraison de projets impliquant agences de développement internationales, ONG et entreprises étrangères, sur fond de clientélisme centralisé aux plus hauts niveaux de l'Etat et de décentralisation ambiguë (Rusten et al., 2004). Le champ politique national est par ailleurs structuré par un parti hégémonique qui n'a pas hésité à plusieurs reprises (en 1992/93, en 1997 et sporadiquement en 2001/02) à recourir à des méthodes violentes de répression de la dissidence politique ou syndicale (Bertrand, 2004 ; Bayart, 2004 ; Vickery, 2007). Le projet que nous étudions est un projet de réhabilitation des polders Prey Nup non loin du principal port du pays (Sihanoukville) On verra qu'il tente de construire une relation au contexte local et aux politiques publiques dans un temps moins heurté et plus prévisible que celui que proposent à la fois le régime et les bailleurs internationaux, dans une logique politique d'institutionnalisation du dispositif mis en œuvre. 


\section{Projet pilote, transfert de gestion et participation}

Le projet de réhabilitation des polders de Prey Nup démarre en janvier 1998 au sud du Cambodge, près de Sihanoukville, exactement là où la mère de Marguerite Duras s'est battue contre le Pacifique (Duras, 1950) ${ }^{11}$. Les travaux sont réalisés par l'entreprise cambodgienne de travaux publics Seng, le contrôle des opérations par Handicap International (HI), tandis que l'appui au développement est confié au groupement constitué par $\mathrm{HI}$ et le Gret. En 2001, le projet Prey Nup devient officiellement une " expérience pilote " de transfert de gestion de l'eau à une communauté d'usagers des polders (CUP) reconnue la même année par le ministère des Ressources en eau (MREM) : c'est une première pour ce ministère. Le projet est organisé en cinq objectifs (Kibler et Perroud, $2003: 53$ ) : (1) la réhabilitation physique d'aménagements hydrauliques permettant de protéger 11000 hectares de terres rizicoles des intrusions d'eau salée, de gérer la lame d'eau douce dans les polders et de faciliter la circulation dans le périmètre ; $(2)$ le transfert de gestion des polders à une " communauté d'usagers des polders " qui mènera à une gestion plus efficace et pérenne des aménagements, tout en diminuant le poids des charges récurrentes assumées par l'Etat ; (3) l'établissement d'un plan foncier des polders, qui permettra de calculer le montant des redevances et préparera la régularisation des droits de propriété sur les terres ; (4) la mise en place de mécanismes d'appui à la production agricole ; (5) et celle d'un service de crédit rural, qui favorisera l'intensification et la diversification des exploitations familiales.

\footnotetext{
${ }^{11}$ Les polders sont délimités par la digue reconstruite pour une large part sur la base du tracé de l'aménagement colonial des années 1930 d'une région de frontière, pratiquement " vide " à la fin du $19^{\mathrm{ème}}$ siècle du point de vue de la construction et de l'ancrage territorial d'un Etat cambodgien colonial ou indépendant. Cette section s'appuie sur les résultats d'une mission de recherche effectuée en 2005 (Le Meur, Bayart et Bertrand, 2006), utilisant aussi comme source les documents de capitalisation de cette expérience produits par le Gret (Kibler et Perroud, 2003 ; Brun et Fontenelle, 2006). Le projet, financé par l'AFD durant la période 1998-2008, s'est terminé en février 2008 par la signature d'un accord cadre (Memorandum of Understanding) entre le ministère des Ressources en eau et de la Météorologie (MREM), la Communauté d'usagers des polders (CUP) et les autorités locales. Cette période n'est pas traitée dans le présent texte (voir Mounier, 2008, pour une évaluation filmée du projet ; et les commentaires de Fontenelle et Tissier, 2013).
} 
Ces cinq objectifs a priori complémentaires ne sont pas rassemblés en un but commun explicite - accroissement de la production rizicole, augmentation des revenus, lutte contre la pauvreté... - celui-ci étant subsumé a posteriori sous le label suffisamment neutre et consensuel de "développement agricole et rural de la région " (ibid.), correspondant de facto à la notion de " projet de développement intégré ». Ce sont les objectifs 2 et 3 qui vont donner au projet son identité et son caractère " expérimental ${ }^{12}$ puis "pilote ", alors même qu'ils n'étaient pas clairement affichés au démarrage de l'action. C'est donc l'absence de cadre légal (en matière de gestion de l'eau et de foncier) qui qualifie en quelque sorte après coup le projet comme opération expérimentale aux yeux de ses promoteurs. Sa caractérisation comme projet pilote est en même temps vue comme résultant de la "résonance nationale " que le projet acquiert peu à peu en matière de contribution à l'élaboration de politiques publiques dans ces domaines. Le thème du projet pilote est donc ici constitutif du fonctionnement de l'intervention et de sa légitimation au-delà de ses limites physiques; cela vaut tant pour le projet (et donc l'opérateur Gret qui conduira ce projet pendant dix ans au lieu des deux années initialement prévues), que pour la CUP qui signera un accord cadre avec le MREM en 2008, et l'AFD, qui s'appuiera sur ce projet pour nouer une alliance avec la Banque asiatique de développement. En même temps, la spécificité technique du projet - il s'agit de polders, pas d'irrigation - en fait un projet pilote un peu particulier dans le domaine des aménagements hydro-agricoles, rendant le label de « projet pilote » encore plus ambigu.

Ce label aide à la légitimation de l'intervention au-delà de son périmètre d'intervention, comme contributeur potentiel à la construction d'une politique de l'eau, donc dans une logique d'extension et de consolidation du réseau qu'il (et qui le) constitue. En même temps, l'histoire du projet est marquée par la nécessité de se construire comme isolat, ce que la configuration physique particulière des polders facilite a priori. II s'agit cette fois de limiter l'extension du réseau, de le clore, pour éviter en particulier un trop fort " encliquage " politique. Ce souci ne s'est toutefois pas

12 Comme le rappelle Jean-Philippe Fontenelle qui a suivi le projet pour le Gret, "malgré l'ampleur de la situation, rien n'était écrit, tout était à inventer. La situation initiale n'avait pas fait l'objet d'étude de faisabilité détaillée » (Fontenelle et Tissier, 2013 : 3). 
traduit par un retrait passif hors des arènes locales et supra-locales, il a au contraire impliqué un travail actif de négociation d'une position particulière, travail que l'on peut qualifier de " politique de l'apolitisme " : savoir nouer des alliances, construire des compromis, sans se laisser (trop) instrumentaliser par telle faction ou tel parti. Les frontières du projet sont de facto poreuses et changeantes. L'économie interne de la Communauté des usagers des polders (CUP), les polders dont elle a la gestion sont tributaires de l'environnement local immédiat (zone de mangrove les bordant, piémont à l'ouest de la route nationale 4, Parc national de Ream), de la municipalité de Sihanoukville et de la scène nationale. L'extension des polders a en outre varié dans le temps, et la perméabilité de leurs frontières (par rapport en particulier aux usagers que sont les pêcheurs) est sujette à des interprétations variables. Les polders constituent, du point de vue des usages aussi bien légaux et officiels qu'illégaux et officieux, un espace sociopolitique à géométrie variable. Ils font l'objet d'un travail collectif et incessamment contesté de définition de leurs limites et de la légitimité de celles-ci, ce qui vaut aussi pour la CUP (cf. infra). La construction d'un dispositif sociotechnique obéit à la règle qui veut que :

"[...] les objets techniques définissent dans leur configuration une certaine partition du monde physique et social, attribuent des rôles à certains types d'acteurs - humains et non-humains - en excluent d'autres, autorisent certains modes de relation entre ces différents acteurs, etc. » (Akrich, 1987 : 49).

L'implication d'acteurs a priori extérieurs au projet (appartenant au champ politique et administratif) cristallise la tension suivante: des acteurs individuels sont les artisans d'une institutionnalisation qui suppose et produit un dépassement des facteurs personnels. Le projet produit un espace d'intéressement qui englobe mais dépasse sa seule utilité technique. Parce qu'il génère un domaine de ressources confié à des personnels dotés de compétences spécifiques, sa défense (au prix de la reformulation progressive de ses objectifs) devient un argument majeur dans des luttes de prestige et d'influence internes aux bureaucraties et aux instances partisanes. Les logiques d'intéressement accroissent la polysémie du projet. Tel fonctionnaire du MREM ou du cadastre municipal jouera le jeu de sa promotion non pas tant parce qu'il est convaincu des vertus démocratisantes des dispositifs participatifs qu'en raison de ses intérêts directs de carrière. Le caractère de « projet pilote » des polders de Prey Nup 
accentue un peu plus encore la productivité politique de ces effets d'intéressement : des responsables des départements techniques municipaux de Sihanoukville organisent des visites guidées du site à l'intention de leurs collègues originaires d'autres provinces, manière de se valoriser en s'identifiant à un objet de réussite technique. Du point de vue de la politique du projet, on peut formuler la contradiction ainsi : s'appuyer sur des personnes clefs pour enclencher un processus visant à s'extraire d'une dépendance vis-à-vis de ces personnes. On se situe dans le cadre d'une reconnaissance pratique et d'une volonté de dépassement du développement comme clientélisme, et donc dans un projet d'institutionnalisation, sans que cela soit énoncé comme tel.

La gestion de cette tension est centrale dans la stratégie d'inscription de la CUP dans la durée. Que ce soit par rapport aux conflits fonciers ou au suivi des mutations de parcelles, elle obéit à une logique fonctionnelle tout en se gardant d'apparaître comme l'instrument de l'Etat, voire son représentant. La négociation d'une alliance avec les communes pour le recouvrement de la redevance s'accompagne d'une nécessaire prise de distance face à des enjeux sensibles comme la question foncière. En même temps, le fait de prendre en charge le suivi des mutations foncières (objectif 3 du projet) s'apparente à la reprise d'une fonction étatique (c'est la compétence du Cadastre) par un organisme non étatique au statut associatif et (encore) lié à un projet de développement dont les opérateurs sont des ONG étrangères.

Aux notions de participation et de projet pilote s'ajoute celle de transfert dans la construction par le projet d'un discours légitimant et l'enrôlement d'alliés. La politique officielle du gouvernement cambodgien en matière d'aménagements hydro-agricoles s'inscrit dans une optique explicitement participative, impliquant la reconnaissance de "communautés d'usagers de l'eau ${ }^{13}$. La notion de transfert de gestion est au cœur du

13 Malgré cette orientation du gouvernement, des modèles concurrents de gestion de l'irrigation peuvent être soutenus par certains secteurs politico-administratifs : c'est l'un des défis auquel est confronté le dispositif articulé autour de la CUP qui prévaut à Prey Nup, et dont la pérennisation pourrait être mise en échec par d'autres modèles (par exemple la régie directe), et donc par d'autres bailleurs de fonds (par exemple la Corée du Sud ou la Chine) (voir Le Meur, Bayart, Bertrand, 2006 : 18-19, 34-35 ; voir aussi AFD, 2001/2005, concernant 
dispositif mis en place par le projet et de ses objectifs de durabilité (mais $50 \%$ de cette maintenance est financée par le ministère). L'objectif de transfert de gestion de l'Etat vers les usagers des polders structure le discours et les actions du projet de Prey Nup. L'idée paraît simple. Elle correspond en outre pleinement à la rhétorique actuelle d'empowerment, de décentralisation politique et administrative, de participation populaire. En même temps, la notion suppose un émetteur, un récepteur, un opérateur et un objet de transfert. A y regarder de plus près, l'impression d'évidence se trouble. Ce point a d'ailleurs été identifié par la coordination du projet :

"Il ne s'agit pas de transférer la gestion à une société paysanne ayant une expérience historique de la gestion de l'eau et des problèmes d'action collective que cela entraîne, mais bien de créer de telles capacités, dans un contexte où le cadre légal et institutionnel est fragmentaire. » (Kibler et Perroud, 2003 : 210)

Identifier une question ne veut pas dire en tirer toutes les implications. Premier point: l'objet à transférer est de fait entièrement créé par l'opération, en l'occurrence des infrastructures physiques et un ensemble de règles et de procédures devant permettre leur gestion. Second point découlant du précédent : l'Etat n'a de ce point de vue rien à transférer. Le caractère informel de la délégation de la gestion des polders à la CUP et le label de "projet pilote " expriment nettement ce fait ${ }^{14}$. En même temps, cette délégation " existe " au sens d'une délégation par le gouvernement d'un espace défini (les polders) selon une logique de "décharge ", de " gouvernement indirect » à la nature hybride et aux modalités non encore stabilisées. Pourquoi parler de "transfert " dans ce cas ? La réponse de l'équipe du projet apparaît radicalement pragmatique : "La question du 'contenu' du transfert se précise au fur et à mesure de l'émergence de la CUP » (ibid. : 114).

les réflexions de l'Agence française de développement en la matière à cette époque). Ceci explique aussi l'importance du projet pour l'AFD et son alliance avec la Banque asiatique de développement, bien documentée dans le film de Mounier (2008).

${ }^{14}$ La signature en 2008 d'un Memorandum of Understanding entre la CUP et le ministère (voir note supra) marquant la fin officielle du projet n'infirme pas véritablement ce constat, dans la mesure où elle vient légitimer ex post un processus de construction sociotechnique dont la CUP et les polders constituent des acteurs structurants. 
Elle laisse pourtant de côté ce qui est peut-être l'essentiel, à savoir la fonction du discours du transfert de gestion. Ce discours fonctionne comme un opérateur de traduction des intérêts hétérogènes des acteurs individuels et collectifs impliqués dans cette entreprise : équipe du projet, Gret, représentants de la CUP, usagers, autorités locales, MREM, Veng Sakhon (chef de projet, personnage haut-placé du ministère et du Parti du peuple cambodgien, le PPC), le premier ministre Hun Sen, l'AFD. II crée une mobilisation et un intéressement et facilite ainsi la construction d'alliances permettant au projet d'avancer en se consolidant. Ce sera en particulier l'enjeu du rapprochement de l'AFD avec la Banque asiatique de développement, stratégique pour l'AFD qui utilisera les réussites de Prey Nup pour construire cette alliance réinvestie dans des projets ultérieurs ${ }^{15}$.

Le discours du transfert a toutefois ses propres faiblesses. Tout d'abord, l'alliance nouée entre les acteurs n'est pas complètement consolidée du côté des usagers. Le degré d'appropriation de la CUP par ses membres n'a pas été évalué ${ }^{16}$, mais la proportion d'impayés, qui s'établit autour de $15 \%$, ne constitue pas un indicateur probant d'une faiblesse de cette appropriation. Or l'adhésion des populations ne passe pas forcément par le discours du transfert (qui serait à usage externe) mais plutôt par la qualité du service offert, et peut-être plus encore par l'évidence des résultats en termes de production rizicole. Second point : le discours du transfert pourrait être supplanté par celui de la décentralisation (de la reconstruction de l'Etat, de la fiscalité) comme moteur des alliances et des intéressements. Un tel changement risquerait de placer le projet et plus tard la CUP en porte-à-faux, quel que soit son mode "réel » de fonctionnement sur le terrain. L'autonomie de la CUP, sa "politique de l'apolitisme » risquent d'être hypothéquées par le processus de décentralisation (Rusten et al., 2004) qui pourrait pousser les autorités locales et communales à affirmer leur prééminence, voire à remettre en cause la délégation de la

${ }^{15}$ Voir à ce sujet les interviews d'Hervé Conan (AFD) et Alain Goffeau (BAD) dans Mounier (2008).

16 Il semble assez faible, si l'on en croit les conclusions d'une enquête sur le thème du (non) paiement de la redevance sur l'eau, réalisée en 2006 par Alban Guillemet dans le cadre du master « Anthropologie et métiers du développement durable » de l'université de Provence. 
gestion de l'eau à une association d'usagers dont la redevance entre en concurrence avec la participation financière demandée aux villageois pour les projets de développement communal, dans le nouvel environnement politique qu'ont créé les premières élections locales (février 2002) et le lancement du Programme SEILA (Social Economic Improvement Local Agency). D'ores et déjà, les responsables administratifs chargés de ce programme à la municipalité de Sihanoukville ne font pas mystère de leurs interrogations quant à un transfert des fonctions de la CUP aux communes, même s'ils le jugent grandement prématuré dans l'état actuel des compétences locales et se félicitent de l'œuvre accomplie. Ils pourront trouver quelque inspiration dans l'effort que le PNUD et la Commission de I'Union européenne entendent consentir en faveur de l'intercommunalité ${ }^{17}$.

Le cas de figure dans lequel on se trouverait alors ressemble à la situation décrite par David Mosse dans son analyse d'un projet de développement agricole en Inde, et dont il extrait l'une de ses cinq propositions générales :

"Projects do not fail ; they are failed by wider networks of support and validation. [...] project failure is not the failure to turn design into reality; but the consequence of a certain disarticulation between practices, their rationalising models and overarching policy frameworks. Failure is not a failure to implement the plan, but a failure of interpretation. » (Mosse, 2005 : 18)

L'assemblage d'éléments hétéroclites au principe de l'intervention de développement et de la construction de politiques publiques est un travail continué, dont l'issue reste ouverte, le succès ou la mise en échec dépendant du type d'extension et de la solidité du réseau sociotechnique qui constitue et institue le projet comme objet d'intéressement pour des acteurs suffisamment influents (leur influence faisant elle-même l'objet de retournements du même ordre).

\footnotetext{
${ }_{17}$ Sur ce point voir les commentaires de Jean-Philippe Fontenelle (GRET à l'époque) et José Tissier (AFD), respectivement : « De mon point de vue, la CUP s'oriente progressivement vers des prémisses de gestion intercommunale d'un service collectif »; et: "L'évolution de la relation de la CUP avec les autorités et collectivités locales pourrait devenir source de conflit, notamment si dans le futur ces dernières détiennent des pouvoirs effectifs d'imposition " (Fontenelle et Tissier, $2013: 5$ ).
} 


\section{L'œuvre de concepts : village, usager, communauté}

L'intervention mobilise dans sa construction des concepts qui sont souvent piochés dans le stock des théories obsolètes en sciences sociales pour connaître une seconde vie comme infra-idéologies sous-jacentes aux politiques de développement. Les notions de village et de communauté occupent à cet égard une position ambiguë dans l'exemple cambodgien de Prey Nup.

L'échelon villageois constitue un élément certainement pas impensé, mais à tout le moins non discuté du projet, et pourtant il est présenté comme essentiel : "L'importance de l'espace villageois » (Kibler et Perroud, 2003 : 93) est soulignée dans le diagnostic rapide des polders réalisé en 1998 et l'assemblée villageoise est présentée comme "l'espace de discussion et de propositions " (ibid. : 94), sans plus d'explication. Le point mériterait pourtant d'être étayé, et ce de deux points de vue. Sur le plan pratique du fonctionnement de la CUP, l'existence d'un échelon local doté d'une certaine consistance sociale et politique au-delà du seul découpage administratif peut aider à l'ancrage du dispositif. De fait, le choix a été fait de privilégier ce niveau avec la mise en place de responsables villageois chargés en particulier de collecter la redevance sur l'eau pour la CUP. D'un point de vue plus théorique, l'assertion de la force de l'échelon villageois va à l'encontre des thèses que l'on peut trouver dans la littérature sur « le village cambodgien ", littérature qui souligne plutôt la faible consistance du village comme espace politique et lieu des solidarités " horizontales" et la prégnance des relations clientélistes "verticales " (Népote, 1992; Ovesen et al., 1996 ; Ledgerwood et Vijghen, 2002) ${ }^{18}$. Il faut en outre inscrire la discussion dans le contexte post-Khmers rouges. L'administration, sous influence vietnamienne, s'est lancée dès 1979 dans une stratégie de promotion des formes coopératives ( $k r o m$ samaki) et de l'économie familiale relevant d'une politique de collectivisation mais aussi de villagisation énergique (Slocomb, 2003 : 96-111) qui est actuellement rejetée.

18 Cette littérature ne s'éloigne toutefois pas systématiquement d'une "infra-idéologie » (Olivier de Sardan, 1995 : 59) communautaire qui continue d'imprégner les pratiques et représentations développementistes au Cambodge comme ailleurs (voir Ovesen et al., 1996 : 69 ; Vijghen et Sareoun, 1996). 
Les sens du mot village et ses usages par le projet dans le contexte des polders de Prey Nup sont sources de frictions. Ceux de communauté aussi. Le terme charrie une longue histoire qui imprègne le monde et le langage du développement (Chauveau, 1994 ; Agrawal et Gibson, 1999 ; Sivaramakrishnan, 2000 ; Le Meur, 2008b). La communauté avait été initialement imaginée comme l'échelon pertinent de l'intervention (les ambiguïtés de la notion sont discutées infra), avant que ne s'impose en 2001/02 l'idée d'une cogestion et d'un transfert. Et encore cette idée ne s'est-elle imposée que très progressivement, via une "institutionnalisation de l'ambiguïté » (Le Meur et al., $2006: 25$ ) quant à la nature et aux prérogatives de la CUP et à la question spécifique du prélèvement d'une redevance permettant de garantir le fonctionnement et la maintenance des infrastructures du projet. La redevance constitue en soi un élément hybride, un pivot de l'ensemble du dispositif. Les débats internes au projet se sont essentiellement concentrés sur les procédures techniques et institutionnelles, sur la question du recouvrement et des impayés, et la fixation du niveau annuel de prélèvement, sans doute aux dépens d'une discussion sur sa nature et l'enjeu intrinsèque qu'elle représente, du fait de l'ambiguïté de son statut institutionnel.

Tout d'abord, la redevance sur l'eau est plus qu'une redevance sur l'eau, puisque les contreparties en sont multiples et qu'elles se sont dessinées au cours du projet : accès à l'eau, maintien de l'infrastructure, dispositif d'enforcement, légitimation des droits fonciers... Ensuite, elle s'inscrit a priori dans le champ sémantique structuré par les notions de développement, société civile ${ }^{19}$, participation et contribution, par opposition au domaine de l'Etat. La première raison à cela est stratégique, de démarcage par rapport à l'Etat, et l'équipe du projet fait attention aux mots employés,

\footnotetext{
19 La notion de "société civile " est ici entendue au sens du " troisième secteur " associatif, représenté en l'espèce transitoirement par l'équipe du " projet » qui doit préparer le terrain et céder la place à la CUP comme émanation " locale "; voir Salemink (2006) sur les ONG étrangères comme produit de substitution à la société civile dans le contexte vietnamien, configuration qui vient nuancer la présentation de Lewis qui note un remplacement de la notion d'ONG par celle de société civile dans les mots clefs du développement des années 1990 (Lewis, 2009 : 39-40).
} 
évitant toute connotation fiscale ${ }^{20}$. Le projet se situe ici dans une perspective fonctionnelle caractéristique de la logique localisée du développement. La redevance est prélevée sur les "usagers", les "populations cibles " du projet, selon un objectif direct de financement de prestations en direction de ces mêmes usagers. Le niveau géographique de péréquation est constitué par l'ensemble des six polders et la part de la redevance qui va aux communes n'est quant à elle pas réinjectée dans le budget communal. Elle sert à rémunérer l'activité des élus et des policiers communaux impliqués dans le recouvrement des impayés. II s'agit de facto de financer leur participation à la gestion des polders.

L'interprétation de la redevance comme contribution est pourtant insuffisante, dans la mesure où la CUP prend en charge des fonctions qui relèvent du domaine de l'Etat, et dans la mesure également où elle est contrainte de faire appel à l'Etat pour réaliser certains de ses objectifs fonctionnels (le recouvrement des impayés et la sanction des usages déviants, qui impliquent à certains moments l'intervention, plus ou moins légitime, des forces de police communales). On peut voir l'ensemble du dispositif sous l'angle de la délégation dans une logique concessionnaire ou comme un effet de " privatisation de l'Etat ». On peut aussi insister sur la délivrance d'un service collectif au cœur du projet de la CUP, qui de fait différencie la situation de celle des concessions forestières ou foncières attribuées en toute opacité et sans souci du bien public (United Nations, 2004 ; Hibou, 2004). Pour interpréter la nature de la redevance, il faut la traiter en lien avec les relations de propriété réglant les rapports aux différents éléments des polders. C'est l'Etat, via le ministère des Ressources en eau (MREM), qui est le propriétaire des aménagements, et en particulier de la digue principale. Nous sommes donc en présence d'une infrastructure publique contribuant à la délivrance d'un service à un collectif d'usagers. On peut considérer que le paiement de la redevance s'inscrit de ce point de vue dans une logique fiscale ou parafiscale, d'où découle un sentiment de sécurisation des ressources sur lesquelles on travaille.

${ }^{20}$ C'est aussi l'argumentaire retenu par le premier ministre Hun Sen dans le discours de soutien à la politique de transfert de gestion qu'il a tenu à Prey Nup en 2003. 
En revanche, la terre, qui constitue le support des aménagements et des usages des polders, devrait passer en pleine et entière propriété des détenteurs de titres - qui ne sont pas systématiquement les usagers - une fois achevée l'opération d'immatriculation foncière. Il s'agit également d'une opération pilote, destinée à s'insérer dans le programme national dont le LMAP (Land management and administration program), financé par la Banque mondiale, est l'opérateur. Pour le projet Prey Nup et la CUP, la question foncière apparaissait initialement comme un enjeu fonctionnel, une affaire d'identification des individus soumis à la redevance foncière. Elle est devenue autre chose, à mesure qu'avançait la réflexion sur le dispositif institutionnel dans un contexte juridique toujours incertain. La propriété foncière $s^{\prime}$ inscrit ici dans le triangle constitué par les catégories d'usager, de membre de la CUP et de propriétaire foncier. La prestation de service (gestion de l'eau et des infrastructures) est destinée aux usagers de cette prestation qui, en contrepartie, s'acquittent d'une redevance. Ce schéma d'échange direct de prestation et contre-prestation butte sur I'inachèvement du cadre légal qui vient compliquer la situation du point de vue de l'application et de la sanction (enforcement).

La circulaire $n^{\circ} 1$ prévoit en effet, sur la base du droit associatif civil, que l'adhésion à une association paysanne des usagers de l'eau doit être volontaire. Cela signifie qu'il est impossible de forcer de quelque façon les usagers à être membres de la CUP. Dans le cadre du droit civil cambodgien - qui est de nature essentiellement jurisprudentielle, donc contradictoire, et qui, de plus, n'est pas borné par un système d'appel en bonne et due forme - une association, fût-elle d'usagers, n'a de légalité que si elle permet, de par ses statuts, de ne pas en être membre. Une association qui contraindrait, par des sanctions ou des incitations négatives, une catégorie de population à devenir ses membres pourrait être poursuivie au civil. Par conséquent, seuls les membres de la CUP sont dans l'obligation statutaire de payer la redevance. II est de même impossible d'isoler un usager mauvais payeur pour le sanctionner en lui déniant l'accès au service. La formule pratique retenue depuis le démarrage du projet ${ }^{21}$ est de $s^{\prime}$ appuyer

${ }^{21}$ " Les 6000 familles ne sont pas toutes 'usagers des polders', et les usages sont divers: agriculture/élevage, pêche, transport, etc. En revanche, les propriétaires de terre dans les polders sont probablement les plus nombreux, les plus motivés et les plus aisément identi- 
sur les propriétaires des parcelles des polders (d'où l'opération cadastrale), et ainsi de faire du cadastre réalisé l'" assiette fiscale " de la redevance, mais cette option n'a pas de base légale ferme et différentes options sont à l'étude (Le Meur et al., 2006 : 28).

On observe donc des glissements sémantiques et fonctionnels entre les notions d'usager, de membre de la CUP et de propriétaire foncier auquel revient in fine la charge de la redevance sur l'eau. Ils renvoient bien sûr à des questions pratiques dont la résolution est indispensable à la survie de l'entreprise de réhabilitation des polders (gestion financière et technique). La mise en avant du propriétaire foncier par rapport à l'exploitant (qui est le bénéficiaire direct du service) se justifie aussi par l'augmentation des prix des terrains occasionnée par la réhabilitation et l'aménagement des polders. Le paiement par le propriétaire correspond ici à une sorte de taxe sur une rente foncière différentielle.

Au-delà des questions pratiques de fonctionnement, on relève un paradoxe dans la dénomination même de la CUP, qui apparaît comme une sorte d'oxymore : les concepts de communauté, d'usager et de membre renvoient à trois registres différents, respectivement (et schématiquement) celui de l'appartenance, celui de la fonction et celui de l'adhésion. Né de la nécessité, ce télescopage sémantique est tout à la fois productif et porteur d'ambiguïtés et de difficultés. N'a-t-on pas finalement affaire à une sorte de "communauté non communautaire " ? D'une part, le terme a été imposé par les nécessités du cadre institutionnel national dans lequel le projet s'inscrivait. Il faut d'autre part se rappeler que les termes khmers dans lesquels ce cadre est exprimé ne correspondent pas nécessairement aux distinctions sémantiques anglophones ou francophones, ni aux débats et aux connotations idéologiques qui leur donnent leur couleur ${ }^{22}$.

fiables via leurs parcelles : on peut faire le choix d'engager la discussion avec eux dans un premier temps, les autres usagers se joignant plus tard au processus. " (Kibler et Perroud, $2003: 93)$

${ }^{22}$ La CUP n'est le support d'aucune sociabilité particulière, par exemple en termes de funérailles ou de banquets, ce qui est, somme toute, assez remarquable. La restauration de la Fête des Eaux à Prey Nup ne contredit pas cette observation car cette dernière n'est pas organisée par la CUP, même si celle-ci aligne un bateau, mais par les autorités locales qui ont emprunté l'idée au GRET. 
"Le terme de 'communauté" n'a été choisi ni par le projet ni par les usagers. On ne peut pas dire qu'il nous plaise. D'ailleurs, le projet parlait initialement d'une 'association des usagers des polders'. Si c'est finalement le terme de 'communauté' qui s'est imposé, c'est que c'est celui qui est utilisé dans les textes officiels du gouvernement (à commencer par la Circulaire $n^{\circ} 1$ ), et dont l'usage est devenu une condition pour l'enregistrement des 'farmer water users communities', suivant la traduction officielle anglaise de l'expression utilisée en khmer. Même si le champ sémantique du terme 'sahakum' en khmer ne se superpose sans doute pas exactement à celui du terme anglais de 'community', lequel n'est pas non plus entendu en anglais de la même manière que le français entend 'communauté'... Toujours est-il que ce terme de 'communauté' est très en vogue actuellement dans le langage politique et juridique pour désigner toutes sortes d'organisations collectives (notamment rurales). Il est vrai qu'en khmer, le choix des termes (communauté, syndicat, association, coopérative, etc.) est non seulement complexe du fait de la sémantique, mais aussi du fait de la charge historique des mots. Tout ça pour dire que le fait que la CUP s'appelle "communauté" ne signifie absolument pas qu'à quelque moment que ce soit, il y ait eu une quelconque volonté de 'mettre en œuvre une régulation "communautaire" et harmonieuse de l'eau et de l'espace' $a u(x)$ sens que nous donnons en français à l'adjectif 'communautaire'. " (J.-M. Brun, commentaire du rapport d'étape FASOPO du 30 novembre 2005)

Cette appréciation émanant d'un insider correspond assez bien au récit fait de la genèse de la CUP que l'on trouve dans le document de capitalisation (Kibler et Perroud, 2003 : 93 et sq.). On observe en particulier une relative absence de réflexion sur le terme de " communauté " et, curieusement, sur la notion d' « usager », au-delà du constat de l'hétérogénéité de la catégorie ${ }^{23}$.

L'épisode de l'oubli des échelles à pirogue - facilitant le passage des bateaux entre les polders et la zone extérieure de mangrove - dans le plan de réhabilitation conçu à partir de missions d'expertise trop hâtives

23 Voir Sivaramakrishnan (1999: 36) qui distingue deux connotations dans le terme de " communauté ", comme " a morally valued ways of life and the constitution of social relations in a discrete geographical setting ". 
(ibid. : 96) est significatif. Les pêcheurs sont-ils des usagers ? La réponse initiale des experts et du projet est implicitement négative. Ce sont les dommages causés par cet oubli qui amèneront sa réparation : " quand une erreur de conception favorise la mobilisation » (ibid. : 94)... Le document revient sur cette erreur :

"En fait, le projet n'a pas échappé aux travers de la procédure classique de décision sur la conception finale des travaux de réhabilitation à réaliser : faible participation des usagers, suppression de travaux pour contraintes budgétaires, absence d'étude détaillée." (ibid. : 96)

Pourtant, la notion d'usager n'est pas débattue ${ }^{24}$, pas plus que celle de communauté : on reste au fond dans une perspective très pratique et fonctionnelle, qui n'est pas illégitime, au risque d'être très partielle et partiale concernant la définition de l'usager.

Le maintien d'ambiguïtés, ou du moins d'un flou définitionnel relatif, a pu s'avérer productif. L'objet CUP est difficile à appréhender et les interprétations locales sont diverses. Les usagers et les usages sont hétérogènes (agriculteurs, pêcheurs, commerçants, transporteurs, agents du projet, fonctionnaires), et il est significatif que le projet se soit rabattu sur une identité foncière, pratique et visible, pour lancer le chantier de la CUP. Après tout, les relations foncières ne reconnaissent et ne distribuent pas seulement des droits mais aussi des identités sociales ${ }^{25}$, et le rapport à la terre (les relations de propriété) constitue un point de médiation pertinent entre la définition d'un groupe et les fonctions assurées par une organisation à base territoriale comme la CUP. Les refus de paiement de la redevance ne sont d'ailleurs pas liés à une mise en cause de ce lien propriétaire/payeur, mais bien plus à des pratiques que l'on peut qualifier

\footnotetext{
${ }^{24}$ Nous avons pourtant fouillé le document de capitalisation; ceci dit, il est possible que l'absence d'index nous ait fait manquer quelque chose.

${ }^{25}$ Comme le note Chris Hann (1998: 5) : "It therefore seems desirable to stretch the definition of property beyond the conventional anthropological formula, which proclaims simply that property relations are social relations. The word 'property' is best seen as directing attention to a vast field of cultural as well as social relations, to the symbolic as well as the material contexts within which things are recognized and personal as well as collective identities made ".
} 
d'opportunistes (assurance de ne pas être sanctionné pour les mauvais payeurs haut placés, attentisme pour les autres) ou liées au manque de moyens pour les plus pauvres. Au contraire, le paiement de la redevance est un moyen contradictoire et contesté de légitimer la possession de la terre, comme on peut l'observer dans la partie du Polder I incluse dans l'emprise du Parc national de Ream et donc exclue de l'immatriculation foncière, ou encore avec le conflit foncier centré sur le village de Bek Krang $\left(\right.$ Polder II) ${ }^{26}$. Ce choix effectué au fond très tôt par le projet en faveur du propriétaire payeur (même s'il manque encore une validation légale) éloigne la CUP de l'idée d'un groupe constitué autour d'usages diversifiés d'un espace commun : l'ensemble constitué par le propriétaire payeur, sa parcelle et la conception qui sous-tend ce choix constitue le " point de passage obligé ", moment de "problématisation " (Callon, $1986: 183$ ) de l'entreprise de traduction constitutive du projet. C'est ce que montre l'anecdote évoquée plus haut de l'oubli des pêcheurs. En même temps, l'impasse faite sur les notions de communauté et d'usager, en faveur d'une approche plutôt technique de gestion de l'eau, offre une marge de manœuvre pour des ajustements pragmatiques (on a finalement construit ces échelles de pêcheurs) tout en présentant l'avantage d'être consensuelle (et les succès en matière de production et de rendements rizicoles valident cette option tout en offrant des arguments sans doute plus décisifs aux politiques soutenant le projet que la mécanique institutionnelle mise en place).

Pour autant, le parti-pris technique de la CUP, pratique dans la gestion quotidienne des infrastructures, constitue peut-être aussi un facteur limitant le développement d'un sentiment d'appropriation ou d'identification de la part de ses membres, qui de fait réagissent plutôt en usagers. Les

\footnotetext{
${ }^{26}$ Le président du Tribunal de Sihanoukville assure que le paiement de la redevance ne vaut pas titre de propriété et est lié au strict usage de l'eau (source: entretien, Sihanoukville, 22 novembre 2005). Brian Rohan, le conseiller juridique de Human Rights in Cambodia Project, serait enclin à partager son interprétation sous réserve d'un examen du dossier (source : entretien, Phnom Penh, 25 novembre 2005). Or, comme nous l'avons vu, le lien strict qu'ils établissent entre paiement de la redevance et usage de l'eau est inexact, dans la mesure où, en principe, la redevance est due par le propriétaire et non par l'exploitant. II n'empêche que sur le terrain cultivateurs et propriétaires usent de cet argument pour faire valoir leurs droits antagonistes (sources : entretiens, Sihanoukville, 16-21 novembre 2005).
} 
points de vue des usagers de la CUP sont diversifiés, renvoyant à plusieurs lignes de clivage: membres/non membres, propriétaires/non propriétaires, genre, appartenance ethnique (au sens large, en relation avec l'histoire du peuplement de la zone : non seulement la prise en compte de la forte minorité Cham mais aussi des Khmer Krom et des différentes " vagues " d'immigration vers Prey Nup), couches sociales (voir l'étude socio-économique de Lagandré, 2007). Par rapport à cette question, il est clair que la dimension temporelle est centrale. Elle est très justement relevée dans le document de capitalisation :

"Une contradiction s'impose cependant à nous. L'appui à l'émergence d'une association d'usagers suppose a priori un rythme lent, celui de la réflexion et des discussions, inhérent à tout processus social. Le délai de deux ans imparti au projet impliquait d'aller vite, au minimum 'pour faire nos preuves' et convaincre le bailleur de prolonger son appui. " (Kibler et Perroud, 2003 : 92)

Ajoutons que le délai de deux ans était celui initialement imparti au projet et donc celui qui a constitué le cadre temporel de l'action des agents du projet. La réponse choisie - mais est-ce vraiment un choix ? est donc d'" aller vite " : la logique des projets (logique de décaissement annuel, de court terme, de fractionnement du temps en "phases", de bornage spatial, conceptuel, temporel) ne peut que prendre le dessus dans un contexte national marqué par le poids structurel de la rente du développement dans l'économie politique. Face à cette contrainte, il ne faut pas "placer la barre trop haut»: I'appropriation de la CUP par ses membres renvoie à un processus d'institutionnalisation inscrit dans un terme plus long que celui du projet (Douglas, 1987). En revanche, l'élaboration d'outils incluant des espaces de débat public et de négociation apparaît comme un objectif important et sans doute plus réaliste, qui a produit des effets positifs en ce qui concerne la consolidation du réseau d'alliance dans lequel le projet et la CUP cherchent à s'enchâsser (Le Meur et $a l ., 2006: 31-34)$.

L'histoire de ce projet, formellement achevée en 2007, et celle, encore en construction, de la CUP, mettent au jour l'œuvre des concepts et la part des flous définitionnels dans la gestion pragmatique du projet comme objet délimité et nœud d'un réseau d'alliances. Les notions de participation, de transferts, de projet pilote, de village, de communauté, d'usager 
sont des actants à l'instar des agents du projet, des politiciens, des représentants de l'Etat et aussi des aménagements hydrauliques, des échelles à pêcheurs et des récoltes de riz. Le temps a été également un opérateur central : le sens de sa circulation, lorsqu'un terme vient donner un sens à la fois rétrospectif et prospectif à une action, tout comme ses accélérations différentielles, selon les logiques plurielles du décaissement de l'aide, du calendrier électoral, des cycles agricoles, de l'institutionnalisation d'un dispositif. Le palimpseste du barrage contre le Pacifique se discerne en transparence dans le dessin des aménagements et, pourtant, l'oubli de cette expérience malheureuse a sans doute permis le déploiement de ce nouveau projet.

\section{Conclusion : quel monde commun?}

La réflexion qui irrigue ce texte et l'étude de cas dont il se nourrit dessinent un monde du développement se construisant de manière apparemment paradoxale, à partir de ses capacités d'oubli, de sa propension structurelle à l'amnésie ${ }^{27}$. L'innovation naîtrait de l'oubli, alors qu'en parallèle (et en contraste) avec cette logique de rupture, des concepts obsolètes ou infra-idéologies persistent avec une étonnante vigueur, souvent insérés dans des récits de politique publique dont la force tient à la capacité de stabilisation de relations causales persuasives et rassurantes - une grammaire au sens de Lemieux (2009). On peut ici revenir à la question posée dans les dernières lignes de l'introduction: quel est le monde commun résultant de ce travail de façonnage de la réalité ? Ou plus précisément: s'agit-il de la construction d'un monde - et dans ce cas commun à qui ? qui émergerait des réinterprétations multiples des enjeux du développement et de malentendus productifs ou de la reconduction d'une nonrencontre, d'une mésentente toujours répétée, que la profusion de récits concurrents symboliserait?

${ }^{27}$ Et d'abord de l'oubli des efforts énormes à accomplir pour qu'un projet devienne réalité, comme le suggère Jean-Pierre Jacob en référence à l'ouvrage d'Albert Hirschman, Development Projects Observed (1967). 
La réponse à cette question ne peut être que contextuelle, située, mais la prendre au sérieux implique de dépasser une application trop simpliste de la sociologie de la traduction aux situations de développement ${ }^{28}$. Les processus de traduction décrits dans ce texte, incarnés ou matérialisés dans des acteurs et porte-parole variés - individus, notions, organisations, etc. - peuvent échouer ou, du moins, pour reprendre l'importante nuance apportée par David Mosse (2005), être mis en échec par d'autres réseaux d'actants et chaînes de traductions, comme le montre bien l'avenir incertain du dispositif mis en place dans le cadre du projet Prey Nup et relayé par l'amorce d'une politique nationale de l'eau.

De manière plus profonde, le monde commun créé par le développement est traversé de malentendus, de discontinuités, de mésententes. II n'est " commun » qu'en ce qu'il est mis en forme dans un cadre discursif communément admis et reformulé à l'occasion (le développement affublé du qualificatif "durable »). Cette mise en forme permet à des intérêts et des visions du monde hétéroclites et mutuellement irréductibles de coexister, le plus souvent dans l'évitement ou l'ignorance mutuelle. Cette cohabitation se déploie aussi fréquemment sous l'égide d'un "virtualisme " confondant abstractions conceptuelles, réalités pratiques et prescriptions ${ }^{29}$, parfois dans une prise de parole nouvelle réclamant une place et une voix dans l'arène, ou encore dans le cadre restreint de rencontres

\footnotetext{
${ }^{28}$ Je remercie ici Jean-Pierre Jacob pour avoir attiré mon attention sur ce point important.

${ }^{29}$ La notion de virtualisme (virtualism) a été développée par Carrier et Miller, deux anthropologues de l'économie, dans les années 1990, à partir du constat d'un processus d'abstraction croissante de l'économie "occidentale ", en référence à la notion de désenchâssement (disembedding) forgée par Karl Polanyi. Ce processus d'abstraction ou plutôt d'extraction d'un domaine d'activité et de réflexion (l'économie, l'environnement, etc.) hors de ses attachements sociaux opère à l'échelon pratique et conceptuel et devient "virtualisme " en acquérant une force prescriptive: "Perceiving a virtual reality [...] seemingly real but dependent upon the conceptual apparatus and outlook that generates it [...] becomes virtualism when people take this virtual reality to be not just a parsimonious description of what is really happening, but prescriptive of what the world ought to be; when, that is, when they seek to make the world conform to their virtual vision. Virtualism, thus, operates at both the conceptual and practical levels, for it is a practical effort to make the world conform to the structures of the conceptual » (Carrier \& Miller, 1998: 2; voir aussi Carrier \& West, 2009, pour une extension de la notion aux dispositifs environnementalistes).
} 
localisées et conjoncturelles, qui ne sont que bien rarement les prémisses d'une mise en commun plus large et partagée.

\section{Bibliographie}

AFD, 2001/2005, Aides mémoires concernant les projets hydro-agricoles, Paris, AFD. AGRAWAL A. et GIBSON C., 1999, "Enchantment and Disenchantment: The Role of Community in Natural Resource Conservation ", World Development, 27 (4) : 629-649.

AKRICH M., 1987, "Comment décrire les objets techniques?", Techniques et Culture, $9: 49-64$.

ARENDT H., 1995, Qu'est-ce que la politique ?, Paris, Seuil.

BAYART J.-F, 2004, Libéralisation politique et violence politique au Cambodge, Rapport de mission, Paris, FASOPO.

BERTRAND R., 2004, Cambodge: le carnaval démocratique, Rapport de mission, Paris, FASOPO.

BIERSCHENK T., 2008, Anthropology and Development. An historicizing and localizing approach, Arbeitspapier, 87, Institut für Ethnologie und Afrikastudien, Mayence, Université Gutenberg.

BIERSCHENK T., ELWERT G. et KOHNERT D., 1993, «Einleitung: Entwicklungshilfe und ihre Folgen ", in BIERSCHENK T. et ELWERT G. (dir.), Entwicklungshilfe und ihre Folgen. Ergebnisse empirischer Untersuchungen in Afrika, Frankfurt-New York, Campus Verlag : 7-39.

BRUN J.-M. et FONTENELLE J.-P., 2006, Retour sur un pari. Acquis et enjeux du projet de réhabilitation des polders de Prey Nup, au Cambodge, Paris, Gret.

BOHANNAN P., 1958, "On anthropologists' use of language ", American Anthropologist, 60 (1):161-163.

CALLON M., 1986, "Eléments pour une sociologie de la traduction. La domestication des coquilles Saint-Jacques et des marins pêcheurs dans la baie de Saint-Brieuc ", L'Année sociologique, $36: 169-208$.

CALLON M., 1999, "La sociologie peut-elle enrichir l'analyse économique des externalités ? Essai sur la notion de cadrage-débordement ", in Foray D. et Mairesse J. (dir.), Innovations et performances, Paris, Editions de I'EHESS : 399-431.

CALLON M., LASCOUMES P. et BARTHE Y., 2001, Agir dans un monde incertain. Essai sur la démocratie technique, Paris, Seuil.

CARRIER J. et MILLER D. (éds.), 1998, Virtualism. A New Political Economy, Oxford, Berg. 
CARRIER J. et WEST P. (éds.), 2009, Virtualism, Governance and Practice. Vision and Execution in Environmental Conservation, New York-Oxford, Berghahn.

CHANDLER D. P., 1991, The Tragedy of Cambodian History Politics, War, and Revolution since 1945, New Haven, Yale University Press

CHAUVEAU J.-P., 1994, "Participation paysanne et populisme bureaucratique. Essai d'histoire et de sociologie de la culture du développement ", in JACOB J.-P. et LAVIGNE DELVILLE P. (éds.), Les associations paysannes en Afrique: organisation et dynamiques, Paris, APAD-Karthala-IUED : 2560.

CHONG D., 2002, "UNTAC in Cambodia: A New Model for Humanitarian Aid in Failed States? ", Development and Change, 33(5) : 957-978.

DOUGLAS M., 1987, How Institutions Think, London, Routledge \& Keegan Paul.

DURAS M., 1950, Un barrage contre le Pacifique, Paris, Gallimard.

FONTENELLE J.-P. et TISSIER J., 2013, « Prey Nup, une communauté autour de l'eau (Cambodge) ", Film documentaire, Journées du Tam Dao 2012, Conférences et séminaires (juillet 2013), Paris : AFD.

FRINGS V., 1997, Le socialisme et le paysan cambodgien. La politique agricole de la République Populaire du Kampuchea et de l'Etat du Cambodge, Paris, L'Harmattan.

GOTTESMAN E., 2002, Cambodia after the Khmer Rouge. Inside the Politics of Nation Building, New Haven \& London, Yale University Press.

HALBWACHS M., 1950, La mémoire collective, Paris, Albin Michel.

HANN C., 1998, "Introduction : The Embeddedness of Property ", in HANN C. (ed.), Property Relations. Renewing the Anthropological Tradition, Cambridge, Cambridge University Press : 1-47.

HANNERZ U., 1987, " The World in Creolization », Africa, 57 : 546-559.

HIBOU B., 2004, Cambodge : quel modèle concessionnaire ?, Rapport de mission, Paris, FASOPO.

HINTON A., 2005, Why Did They Kill? Cambodia in the Shadow of Genocide, Berkeley \& Los Angeles, University of California Press.

HIRSCHMAN A. O., 1967, Development projects observed, Washington D.C., The Brookings Institution.

HOBART M. (éd.), 1993, An Anthropological Critique of Development. The Growth of Ignorance, London, EIDOS-Routledge.

KIBLER J.-F. et PERROUD C., 2003, Vers une cogestion des infrastructures hydroagricoles. Construction associative et réhabilitation de polders : l'expérience du projet Prey Nup au Cambodge, Paris, Editions du Gret.

KIERNAN B., 2002 (1996), The Pol Pot Regime. Race, Power, and Genocide in Cambodia under the Khmer Rouge, 1975-79, New Haven, Yale University Press. 
LAGANDRE D., 2007, Etude d'impact du projet de réhabilitation des polders de Prey Nup (Cambodge), Etudes et Travaux, Série en ligne, 15, Paris, Editions du Gret.

LATOUR B., 1987, La science en action, Paris, La Découverte.

LATOUR B., 1992, Aramis ou l'amour des techniques, Paris, La Découverte.

LATOUR B., 2002, "Et si on parlait un peu politique ? ", Politix, 15 (58) : 143-165.

LATOUR B., 2008, "Pour un dialogue entre science politique et science studies ", Revue française de science politique, 58 (4) : 657-678.

LE MEUR P.-Y., 2006, " Governing Land, Translating Rights. The Rural Land Plan in Benin ", in MOSSE D. et LEWIS D. (éds.), Development Brokers \& Translators. Ethnography of Aid and Agencies, Bloomsfield, Kumarian Press : 7599.

LE MEUR P.-Y., 2007, « Anthropologie et développement : une relation à plaisanterie ? ", in BIERSCHENK T., BLUNDO G., JAFFRÉ Y. et TIDJANI ALOU M. (dir.), Une anthropologie entre rigueur et engagement. Essais autour de l'œuvre de Jean-Pierre Olivier de Sardan, Paris, Karthala : 151-174.

LE MEUR P.-Y., 2008a, "Le développement comme constructivisme. Point de vue anthropologique ", Sociétés politiques comparées, 8 , en ligne: www.fasopo.org/reasopo/n8/societespolitiquescomparees8_article.pdf.

LE MEUR P.-Y., 2008b, « Communautés imaginées et politique des ressources naturelles ", in MERAL P., CASTELLANET C. et LAPEYRE R. (éds.), La gestion concertée des ressources naturelles. L'épreuve du temps, Paris, Karthala : 289-301.

LE MEUR P.-Y., 2011, « Une petite entreprise de réassemblage du monde. Ethnographie et gouvernance des ressources foncières en Afrique de l'Ouest ", Ethnologie française, XVI (3) : 431-442.

LE MEUR P.-Y., BAYART J.-F. et BERTRAND R., 2006, La politique de l'apolitisme. De la mise en œuvre d'un projet à l'élaboration d'une politique sectorielle : processus de construction d'institutions de gestion agricole de l'eau au Cambodge, AFD-Gret-FASOPO.

LEDGERWOOD J. et VIJGHEN J., 2002, " Decision-Making in Rural Khmer Villages ", in LEDGERWOOD J. (éd.), Cambodia Emerges from the Past: Eight Essays, DeKalb, Northern Illinois University, Center for Southeast Asian Studies : 109-150.

LEMIEUX C., 2009, Le devoir et la grâce, Paris, Economica.

LENTZ C., 2006, Ethnicity and the Making of History in Northern Ghana, International African Library 33, Edinburgh, Edinburgh University Press.

LEWIS D. 2009, « International development and the 'perpetual present': Anthropological approaches to the re-historicization of policy ", European Journal of Development Research, 21: 32-46.

LYOTARD J.-F., 1983, Le différend, Paris, Editions de Minuit. 
MOSSE D. 2005, Cultivating Development. An Ethnography of Aid Policy and Practice, London, Pluto Press.

MOUNIER E., 2008, Construire contre les marées. Evaluation filmée du projet Prey Nup, 1998-2008, 58', Paris, AFD, Studio K.

NEPOTE J., 1992, Parenté et organisation sociale dans le Cambodge moderne et contemporain, Genève, Editions Olizane.

OLIVIER DE SARDAN J.-P., 1995, Anthropologie et développement. Essai en socioanthropologie du changement social, Paris, APAD-Karthala.

OLIVIER DE SARDAN J.-P., 2011, " Les trois approches en anthropologie du développement ", Revue Tiers Monde, 168 : 729-754.

OVESEN J., TRANKELL I.-B. et ÖJENDAL J., 1996, When Every Household is an Island. Social Organisation and Power Structures in Rural Cambodia, Uppsala Research Reports in Cultural Anthropology, 15, Uppsala, Uppsala University.

PELS P., 1996, "The Pidginization of Luguru Politics: Administrative Ethnography and the Paradoxes of Indirect Rule ", American Ethnologist, 23 (4) : 738761.

PELS P., 1997, "The Anthropology of Colonialism: Culture, History, and the Emergence of Western Governmentality ", Annual Review of Anthropology, $26: 163-183$.

RANCIERE J., 1995, La mésentente. Politique et philosophie, Paris, Galilée.

RUSTEN C., KIM S., ENG N. et PAK K., 2004, The Challenges of Decentralisation Design in Cambodia, Phnom Penh, CDRI.

SALEMINK O., 2006, "Translating, Interpreting, and Practicing Civil Society in Vietnam: A Tale of Calculated Misunderstandings ", in MOSSE D. et LEWIS D. (éds.), Development Brokers and Translators. The Ethnography of Aid and Agencies, Bloomfield, Kumarian Press : 101-126.

SCOTT J., 1998, Seeing like a State. How Certain Schemes to Improve the Human Condition Have Failed, New Haven \& London, Yale University Press.

SIVARAMAKRISHNAN K., 1999, Modern Forest. Statemaking and Environmental Change in Colonial Eastern India, Stanford, Stanford University Press.

SIVARAMAKRISHNAN K., 2000, "Crafting the Public Sphere in the Forests of West Bengal ", American Ethnologist, 27 (2) : 431-461.

SLOCOMB M., 2003, The People's Republic of Kampuchea, 1979-1989: The Revolution after Pol Pot, Chiang Mai, Silkworm Books.

SPITTLER G., 1981, Verwaltung in einem afrikanischen Bauernstaat. Das koloniale Französisch-Westafrika 1919-1939, Wiesbaden, Franz Steiner Verlag.

UNITED NATIONS, 2004, Land concessions for economic purpose in Cambodia. A human rights perspective, Phnom Penh: Cambodia Office of the High Commissioner for Human Rights, United Nations.

VICKERY M., 2007, Cambodia: A Political Survey, Phnom Penh, Funan Press. 
VIJGHEN J. et SAREOUN, L, 1996, Customs of Patronage and Community Development in a Cambodian Village, Phnom Penh, Cambodian Researchers for Development.

\section{Niviviv}

Pierre-Yves Le Meur est anthropologue à l'IRD (Nouméa) UMR GRED (Gouvernance, risque, environnement, développement) E-mail : pierre-yves.lemeur@ird.fr 
